\title{
Multi-antigen-targeted chimeric antigen receptor T cells for cancer therapy
}

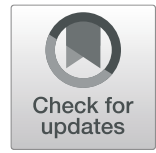

\author{
Xiao Han, Yao Wang, Jianshu Wei and Weidong $\operatorname{Han}^{*}$ (1)
}

\begin{abstract}
The approval of two chimeric antigen receptor-modified T cell types by the US Food and Drug Administration (FDA) for the treatment of hematologic malignancies is a milestone in immunotherapy; however, the application of CAR-T cells has been limited by antigen escape and on-target, off-tumor toxicities. Therefore, it may be a potentially effective strategy to select appropriate targets and to combine multi-antigen-targeted CAR-T cells with "OR", "AND" and "NOT" Boolean logic gates. We summarize the current limitations of CAR-T cells as well as the efficacy and safety of logic-gated CAR-T cells in antitumor therapy. This review will help to explore more optimized strategies to expand the CAR-T cell therapeutic window.
\end{abstract}

Keywords: Chimeric antigen receptor T cells, Logic gate, Antigen escape, On-target, Off-tumor toxicities

\section{Introduction}

Chimeric antigen receptor (CAR)- $\mathrm{T}$ cell therapy has shown dramatic results for hematological malignancies. Recently, two types of CD19-targeted CAR-T cells, tisagenlecleucel (Kymriah), and axicabtagene ciloleucel (Yescarta) were approved by the FDA for the treatment of relapsed/refractory $(R / R)$ B-cell acute lymphoblastic leukemia (B-ALL) and certain types of large B cell lymphoma $[1,2]$.

CAR-T cell therapy represents a landmark in the field of cancer immunotherapy along with immune checkpoint inhibitors, including programmed death-1 (PD-1) and cytotoxic $\mathrm{T}$ lymphocyte-associated protein-4 (CTLA-4) blockade. Patients with a high mutation burden have an ideal clinical response to immune checkpoint inhibitors, whereas patients with low mutation burdens are less responsive [3]. Encouragingly, CAR-T cells have been confirmed to have high efficacy for patients with a low mutation burden [4].

One key to the clinical success of CAR-T therapy is the selection of an ideal target, which should be uniformly expressed on the surface of tumor cells and should not be expressed on vital healthy cells. Despite inducing B cell aplasia, which is clinically manageable, CD19 is considered a promising target based on its

\footnotetext{
* Correspondence: hanwdrsw69@yahoo.com

Molecular and Immunological Department, Bio-therapeutic Department Chinese PLA General Hospital, No. 28 Fuxing Road, Beijing 100853, China
}

expression on B cell malignancies [5]. However, multicenter clinical trials have demonstrated that the relapse rate of CD19-targeted CAR-T cell therapy is approximately $30 \%$ [6], which may be associated with the lack of CAR-T cell persistence and antigen escape, including antigen downregulation or even antigen loss [7]. As an important mechanism for evading CAR-T cell therapy, antigen escape also occurs in non-B cell malignancies as well as in solid tumors $[8,9]$. Another important factor limiting CAR-T therapy is on-target, off-tumor toxicities. The targeting of CD19+ has been shown as a viable strategy, whereas the on-target, off-tumor toxicities from other targeted antigens may be unacceptable or even fatal.

Therefore, the combination of multi-antigen targeting may be a potential strategy to expand the effectiveness of this novel immunotherapy. The Boolean logic gates of "AND", "OR", and "NOT" have been applied to manipulate multi-antigen targeted CAR-T cells to prevent tumor antigen escape and to alleviate on-target, off-tumor toxicities. In this review, we will summarize the limitations of the current clinical applications of CAR-T cell therapy, mainly focusing on the mechanism of antigen escape that leads to disease relapse and CAR-T cell-related on-target, off-tumor toxicities. In addition, we will explore rational strategies to overcome these barriers, thereby increasing the safety and efficacy of CAR-T cells.

(c) The Author(s). 2019 Open Access This article is distributed under the terms of the Creative Commons Attribution 4.0 International License (http://creativecommons.org/licenses/by/4.0/), which permits unrestricted use, distribution, and reproduction in any medium, provided you give appropriate credit to the original author(s) and the source, provide a link to the Creative Commons license, and indicate if changes were made. The Creative Commons Public Domain Dedication waiver (http://creativecommons.org/publicdomain/zero/1.0/) applies to the data made available in this article, unless otherwise stated. 


\section{Limitations of CAR-T cell therapy in clinical application}

Limitation 1: Disease relapse

There are two main forms of disease relapse after CAR-T cell infusions: antigen-positive relapse in the early phase and antigen escape relapse in a later phase. The disease relapse of antigen-positive cells is closely related to CAR-T cell persistence. Factors that affect the persistence of CAR-T cells require further exploration, of which the $\mathrm{T}$ cell-intrinsic quality and CAR structure are critical. Substituting fully humanized single-chain variable fragments (scFvs) for murine scFvs can reduce immune-mediated resistance, thus improving the persistence of CAR-T cells [10]. In the CAR structure, the co-stimulatory signal domain also has an important effect on CAR-T cell therapy. Preclinical and clinical trials have demonstrated that the 4-1BB (CD137) costimulatory domain induced the longer-term persistence of CAR-T cells than CD28 $[11,12]$. Tonic signaling induced by CAR clustering promotes CAR-T cell exhaustion but CD137 costimulatory domain might potentially ameliorate this effect [13]. The CD137, which is significantly different from the CD28 costimulatory domain, has been shown to stimulate fatty-acid oxidation in metabolism and to increase the central memory phenotype, thereby improving CAR-T cell persistence [14].

Antigen escape, including antigen loss or downregulation, is an important mechanism driving disease relapse following CAR-T cell immunotherapy. Alternative splicing is the primary cause of CD19 antigen loss and induces the generation of CD19 isoforms that gradually develop into dominant clusters under the strong immune pressure of adoptive $\mathrm{T}$ cell therapy (ACT), thereby escaping CD19-targeted CAR-T cell-mediated cell death $[15,16]$. Complete antigen loss is not necessary for CAR-T cell resistance, and in some cases, a reduction in antigen density is sufficient to evade CAR-T cells [17]. Nevertheless, a minimum antigen density threshold is required for CAR-T cells to achieve therapeutic efficacy. In preclinical models, Sadelain et al. demonstrated that CAR-T cells extract and transfer target antigens into $\mathrm{T}$ cells via trogocytosis, which leads to a reversible decrease in antigen density. In addition, researchers also verified that combinatorial multi-antigen-targeted strategies could effectively prevent the tumor escape caused by low antigen density that results from CAR-T cell trogocytosis [18].

\section{Limitation 2: On-target, off-tumor toxicities}

Most of the selected antigens are not tumor-specific but are only overexpressed on tumor cells, making it difficult for CAR-T cells to kill targeted tumor cells in a safe manner. B lymphocyte aplasia is a clinically manageable on-target, off-tumor toxicity that is prevalent in the treatment of hematological malignancies with CD19targeted CAR-T cells [19]. However, during solid tumor treatment, on-target, off-tumor toxicities following the infusion of CAR-T cells can occasionally pose serious or even fatal threats [20]. An early clinical trial revealed an unexpected grade 2 to 4 hepatotoxicity after several infusions of genetically engineered $\mathrm{T}$ cells retargeting carbonic anhydrase IX (CAIX) in the treatment of metastatic renal cell carcinoma. Liver biopsies showed that T lymphocytes infiltrated around the bile duct, and there were CAIXpositive bile duct epithelial cells. These observations are sufficient to demonstrate that CAIX-targeted CAR-T cells attack CAIX+ bile duct epithelial cells, resulting in hepatotoxicity [21]. Although target antigens are expressed on both tumor and normal cells, not all kinds of CAR-T cell therapies exhibit observable on-target, off-tumor toxicities, such as targeting carcinoembryonic antigen (CEA), mesothelin (MSLN), and Interleukin 13 receptor, Alpha2 (IL13R $\alpha 2$ ) [22-24]. Furthermore, CAR-T cells targeting the same antigen have demonstrated different on-target, off-tumor toxicities in preclinical trials, some of which were mild, whereas some were lethal, such as targeting fibroblast activation protein- $\alpha$ (FAP) [25-27].

Affinity is another important factor that affects the activity of CAR-T cells. High affinity can cause CAR-T cells to recognize target antigens even at a low density and to proliferate and kill tumor cells, but it can also increase the severity of off-tumor toxicities. However, when the affinity exceeds a certain threshold $\left(K_{\mathrm{d}}<10^{-8} \mathrm{M}\right)$ it makes no significant difference in regard to promoting the efficiency of T cells [28]. Spencer Park et al. demonstrated that micromolar affinity CAR-T cells had stronger antitumor activity and lower off-tumor toxicities than their nanomolar affinity counterparts [29]. Therefore, exploring a rational strategy for regulating CAR-T cell affinity is necessary for controlling the on-target, off-tumor toxicities. It is important to balance the relationship between potency and toxicity in CAR-T cell therapy; therefore, June et al. applied the concept of the "therapeutic window", derived from drug toxicology, to the field of CAR-T cell therapy to achieve the highest therapeutic benefit with acceptable toxicity, thereby expanding and optimizing the clinical application of CAR-T cells in solid tumors [30].

Notably, genetically engineered $\mathrm{T}$ cells, which are mediated by lentiviral vectors or zinc-finger nuclease (ZFN), similar to other genetic engineering therapies, have the potential risks of insertional mutagenesis causing on-tumor, off-target toxicities [31]. In addition, genome-editing techniques have been applied to knock out T cell receptor (TCR) complexes, causing endogenous TCR deficiency in T lymphocytes and avoiding unpredictable off-tumor toxicities [32].

\section{Strategies combining multi-antigen-targeted CAR- T cells}

As antigen escape is the main potential mechanism for evading immunotherapy, the combination of multi- 
antigen-targeted CAR-T cells is being tested as a strategy to reduce relapse [33]. Several major multi-antigentargeted CAR-T cell therapies at present include the following: (I) Pooled CAR-T cells: mixtures of two engineered $\mathrm{T}$ cell lines, each of which expresses a distinct antigen-specific CAR; (II) Dual CAR-T cells: two individual CARs that target different antigens in a single $\mathrm{T}$ cell; (III) Tandem CAR-T cells: two distinct antigenbinding domains are connected in tandem to a single CAR; and (IV) Trivalent CAR T-cells: three CARs target specific antigen molecules in a single engineered $\mathrm{T}$ cell. Based on the multi-antigen-targeting strategy, Boolean logic has been applied to "gate" the activity of CAR-T cells to achieve high efficiency and low toxicity. For instance, $\mathrm{T}$ cells with two independent CAR molecules or a pool mixed with different specific CAR-T cells can use the "OR" logic gate, which enables CAR-T cells to antitumor in the presence of either targeted antigen. However, the "AND" logic-gated CAR-T cells can only be activated in the presence of both antigens simultaneously. In addition, "NOT" logic gate can also be used to make engineered $\mathrm{T}$ cells distinguish target cells from non-target cells, avoiding attacks on normal tissue, thereby achieving the safety of CAR-T cells (Table 1) (Fig. 1, 2).

\section{"OR" logic-gated CAR-T cells for preventing tumor antigen escape Pooled CAR-T cells using the "OR" logic gate}

Pooled CAR-T cells are a mixture of two CAR-T cell lines, each targeting different cognate antigens, thereby achieving lower tumor relapse through an "OR" logic gate. This strategy has been investigated, such as pooling monospecific CAR-T cells targeting human epidermal growth factor receptor-2 (HER2)/IL-13R $\alpha 2$ for glioblastoma and CD19/CD123 for B-ALL [34, 35]. In terms of cytokine secretion and cytolysis, pooled CAR-T cells exhibited lower levels than tandem CAR-T cells and dual CAR-T cells but higher levels than the individual CAR$\mathrm{T}$ cell lines. It is worth noting that the use of two CAR$\mathrm{T}$ cell lines places strong immune pressure on the tumor cells, which may lead to the simultaneous escape of both antigens. In addition, the simultaneous introduction of two CAR-T cell lines may lead to an imbalance in the cell population. The significant amplification of CD19targeted CAR-T cells, which was higher than the amplification of CD20-targeted CAR-T cells, was observed during co-infusion despite the persistence of the CD20 antigen [36].

In addition to the simultaneous mixture of two CAR$\mathrm{T}$ cell lines, a method termed cocktail immunotherapy, which involves the sequential administration of different antigen-targeted CAR-T cells, has also been used in clinical trials. Our team reported a case of a female patient with advanced unresectable/metastatic cholangiocarcinoma (CAA) who was resistant to both radiotherapy and chemotherapy. We successfully infused this patient with epidermal growth factor receptor (EGFR)- and CD133targeted CAR-T cells separately.

The patient underwent two cycles of EGFR-targeted CAR-T cells infusion and achieved 8.5-month of partial remission (PR) until tumor progression was detected by positron emission tomography-computed tomography (PET-CT). Thus, another cycle of EGFR-targeted CAR-T cells combined with anti-PD-1 monoclonal antibody was administration. Subsequent PET-CT revealed newly emerged metastases and previous abdominal tumor enlargement. Since most tumor cells expressed CD133, the patient was enrolled in the clinical trial of CD133targeted CAR-T cell. Radiographic evaluation of metastatic tumors showed a significant reduction or even disappearance with the CD133-targeted CAR-T cell administration, and the patient obtained 4.5-month PR. It is worth noting that both batches of CAR-T cells have caused acute adverse reactions associated with the infusion, among which CD133-targeted CAR-T cell-related acute subcutaneous hemorrhage is serious, requiring clinical emergency intervention [37].

\section{Dual CAR-T cells using the "OR" logic gate}

Dual CAR-T cells are individual $\mathrm{T}$ cells that are engineered to co-express two separate CARs specific for cognate antigens with two individual signals. Depending on the signal transmission pattern, Dual CAR-T cells have various logic-gate operations, including "OR", "AND", and "NOT".

Due to tumor heterogeneity, there are no single specific tumor antigens. Under the strong immune pressure that occurs during immunotherapy, the tumor target antigens tend to selectively escape. This phenomenon is also present in CAR-T cell therapy for glioblastoma (GBM). The combinational multi-antigen targeted strategy may offset the potential antigen escape mechanism. Therefore, Meenakshi et al. generated "OR" logic-gated CAR-T cells that contained distinct CAR molecules targeting two glioma-restricted antigens including HER2 and IL13R $\alpha 2$ (HER2-targeted scFv CD28 $\zeta$ and IL13R $\alpha 2-$ targeted $\mathrm{scFv}-\mathrm{CD} 28 \zeta$ ) [34]. Compared with the pooled populations of HER2-targeted and IL13R $\alpha 2$-targeted CAR-T cells and monospecific CAR-T cells, the HER2"OR" IL13R $\alpha 2$-targeted CAR-T cells more effectively prevented antigen escape and enhanced antitumor efficacy.

This enhancement of antitumor activity may have resulted from the increased $\zeta$-chain signaling by the simultaneous binding of HER2 and IL13R $\alpha 2$. When dual CAR-T cells were exposed to a single HER2 or IL13R $\alpha 2$ antigen, the signal transduction intensity was equivalent 


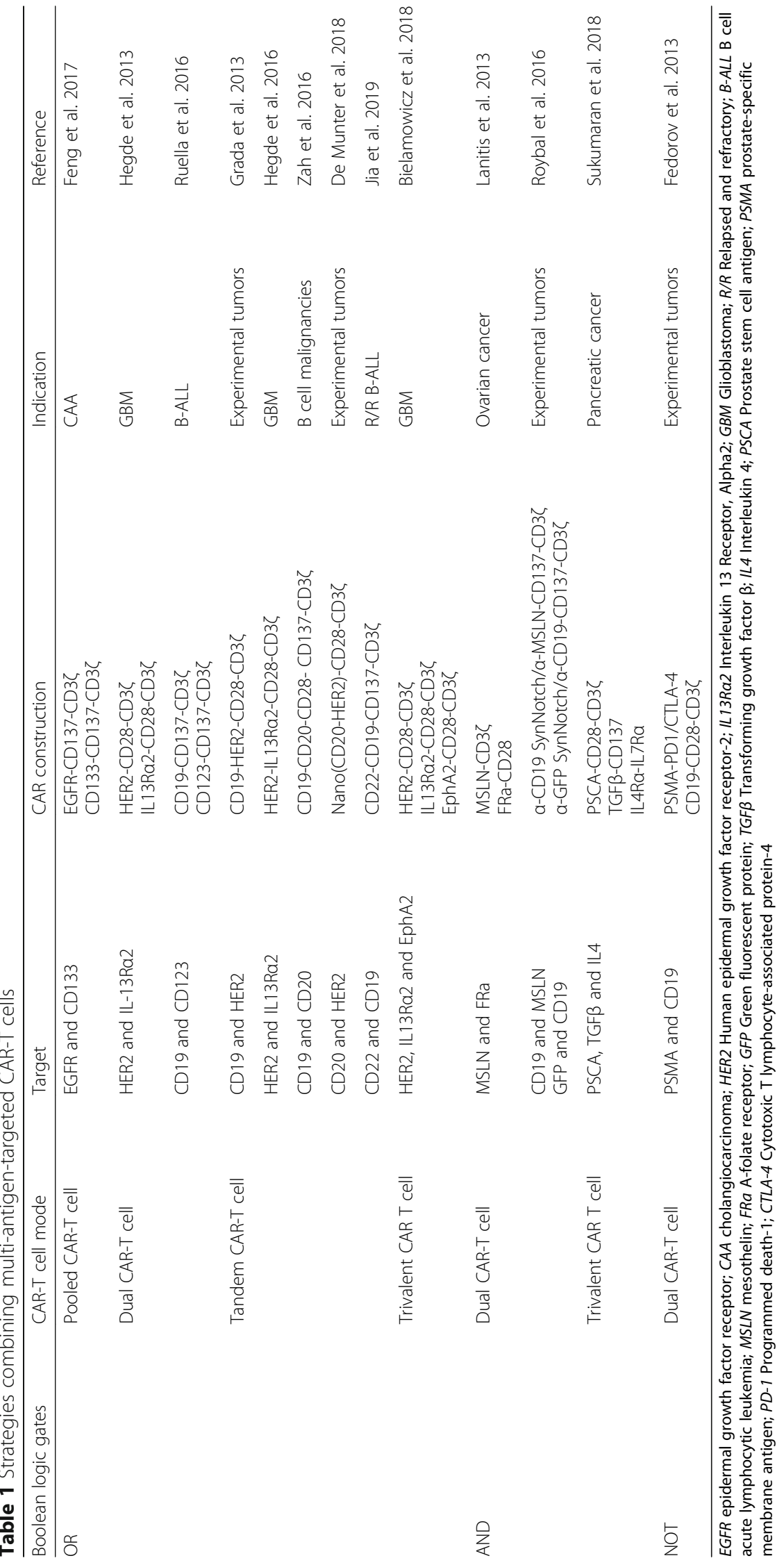




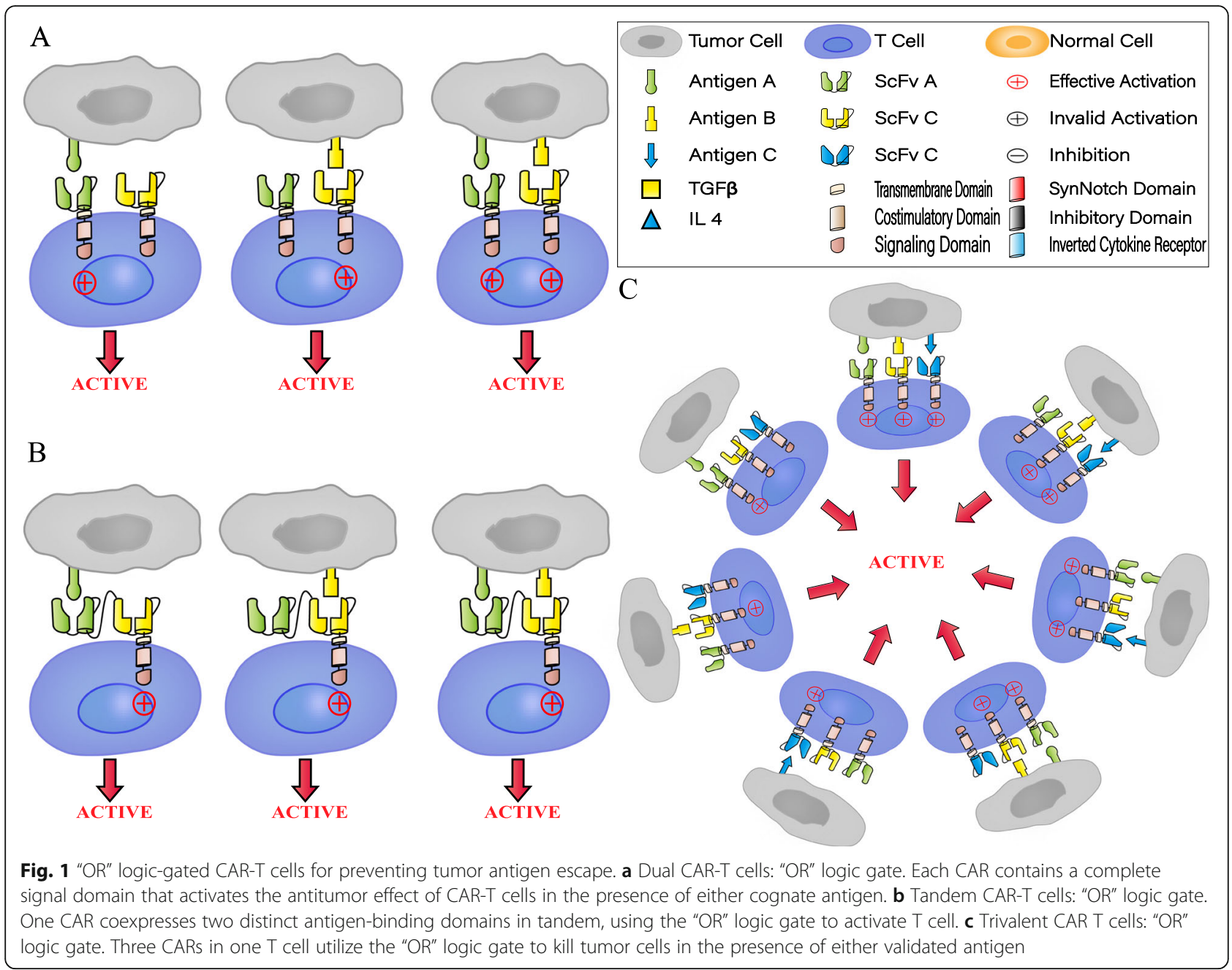

to that of natural $\mathrm{T}$ cells, whereas when dual CAR-T encountered the co-presence of both antigens, maximal downstream signal transduction was exhibited, which was verified by the detection of high levels of zeta-chainassociated protein kinase 70 (ZAP-70) phosphorylation. In addition, studies have confirmed that the density balance between tumor target antigens, and CAR molecules has an important influence on the $\mathrm{T}$ cell response. Dual CAR-T cells are advantageous because they have a highdensity of CAR molecules on the surface of the T cells and target tumor cells with a high density of cognate antigens [34].

CD123 was shown to be highly expressed in most patients with primary B-ALL, especially in patients with CD19-negative relapse following the administration of CD19-targeted CAR-T cells [37]. Based on this phenomenon, effective strategies against antigen escape relapse were devised. Marco Ruella et al. generated CD19- "OR" CD123-targeted CAR-T cells with two complete second-generation CAR structures that included CD19-targeted scFv $\mathrm{CD} 137 \zeta$ and CD123-

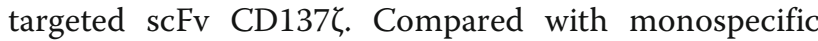
CAR-T cells and pooled CAR-T cells, Dual CAR-T cells exhibited superior antitumor efficacy and longerterm persistence. Interestingly, based on confocal imaging, the two CARs of dual CAR-T cells were involved in the establishment of the same immune synapse with lymphoma cells [35] (Fig. 1a)

\section{Tandem CAR-T cells using the "OR" logic gate}

Tandem CAR-T cells are generated by modifying individual $\mathrm{T}$ cells to co-express distinct scFvs in tandem using the "OR" logic gate. Preclinical trials have provided a proof-of-concept for Tandem CAR-T cells, such as cells simultaneously targeting CD19 and HER2 (CD19/ HER2-targeted scFv- CD28/CD3ל), HER2 and IL13R $\alpha 2$ (HER2/IL13R $\alpha 2$-targeted scFv-CD28/CD3ל) and CD19 and CD20 (CD19/CD20-targeted scFv-CD28/CD137/ CD3ל) $[36,38,39]$. Tandem CAR-T cells caused a distinct response to each of the two cognate antigens, preserving the cytolytic capacity when one of the cognate antigens escaped. In addition, synergistic antitumor 


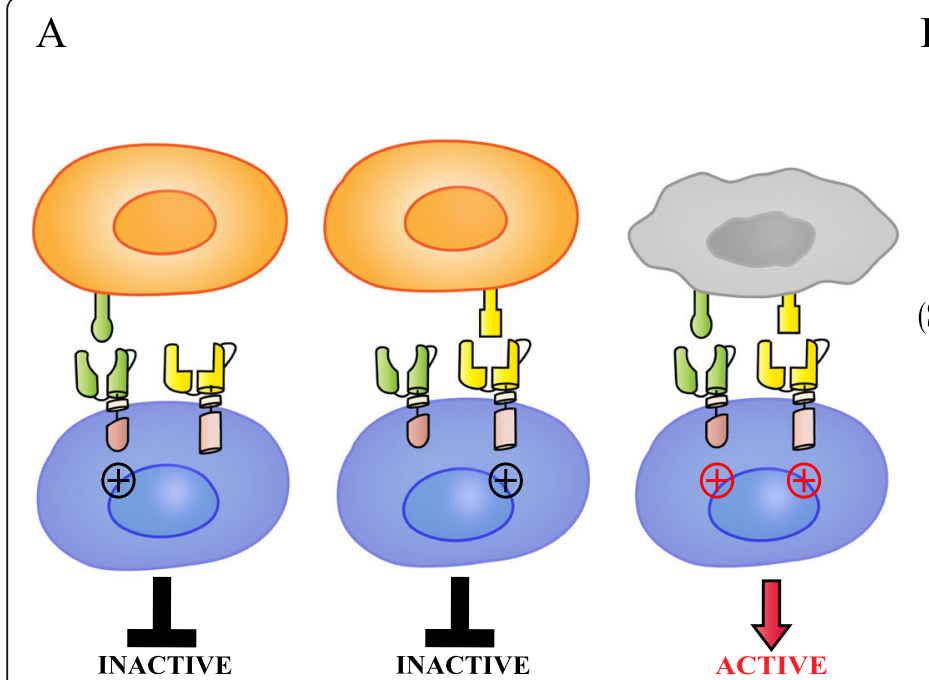

$\mathrm{C}$

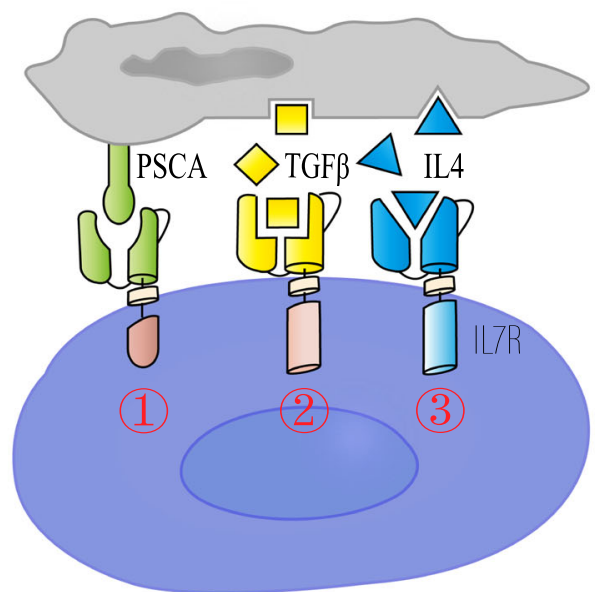

(1) Antigen recognition (2) Costimulation (3) Cytokine release
B

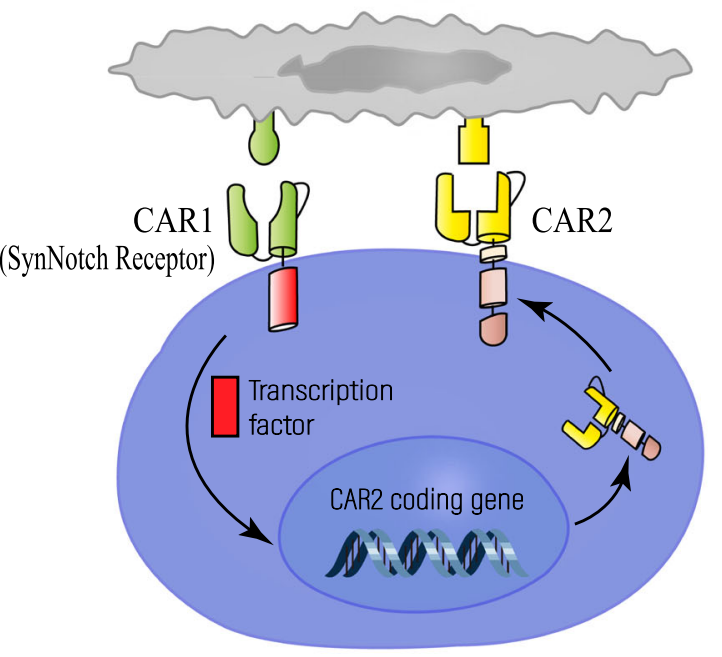

$\mathrm{D}$
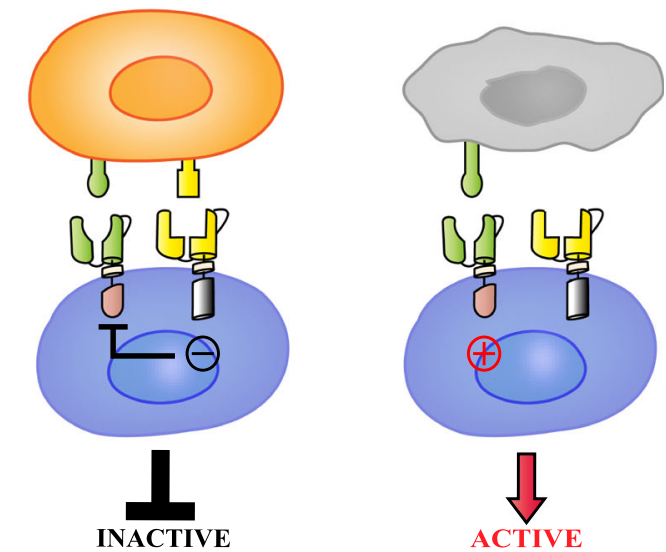

Fig. 2 "AND" and "NOT" logic-gated CAR-T cells for alleviating on-target, off-tumor toxicities. a Dual CAR-T cells: "AND" logic gate. Two distinct CARs are coexpressed with complementary signaling domains in one T cell that fully activates the T cell only in the presence of both cognate antigens. b Synthetic Notch Receptor System: "AND" logic gate. In the presence of cognate antigen of CAR1, SynNotch receptor induces the conditional expression of CAR2 in a transcriptional manner, thereby targeting to the second antigen, and finally achieving highly specific activation of T cell. c Trivalent CAR T cells: "AND" logic gate. Trivalent CAR-T cell response to tumor-specific expression patterns to overcome the immunosuppression of TME, rather than adding additional CARs targeting TAAs. $\mathbf{d}$ Dual CAR-T cells: "NOT" logic gate. iCAR-T cells selectively kill target cells only expressing one antigen, whereas off-target cells co-expressing another inhibitory ligand recognized by iCAR were protected from attack, allowing T cells to distinguish target cells from the off-target cells

efficacy has been demonstrated upon simultaneously encountering both antigens. The formation of bivalent immune synapses in the process of tandem CAR-T cell exposure to tumors co-expressing both cognate antigens is crucial for $\mathrm{T}$ cells to effectively prevent antigen escape and to improve antitumor efficacy [39].

However, some factors have hampered the preparation and application of tandem CAR-T cells, such as the cross-pairing of the variable heavy $(\mathrm{VH})$ and variable light (VL) chains between different scFvs, the limitations of viral vector package sizes, and the immunogenicity of scFvs derived from mice [40]. Fortunately, nanobodies are potential substitutes for scFvs because of their structural specificity, including the absence of light chains, very small structures, mutual noninterference, and weak immunogenicity [41]. De Munter et al. generated and verified nano-based tandem CAR-T cells targeting CD20 and HER2 [42].

Our group reported a clinical case of R/R B-ALL in an adult patient. With the administration of Tandem CAR- 
T cells co-targeting CD22 and CD19 (CD22/CD19-targeted $\mathrm{scFv}-\mathrm{CD} 137 / \mathrm{CD} 3 \zeta)$ after hematopoietic stem cell transplantation (HSCT), more than 14 months of minimal residual disease (MRD)-negative complete remission (CR) was achieved; the patient developed graft-versus-host disease (GVHD), which is clinically manageable. The treatment process of this 22-year-old patient was relatively complicated. He had received a CD19targeted CAR-T cell protocol a month prior to the tandem CAR-T cell protocol, in which CAR-T cells were administered following cytoreduction and lymphodepleting chemotherapy. Although this is a single clinical case, tandem CAR-T cells have exhibited superior persistent remission in the treatment of R/R B-ALL [43] (Fig. 1b).

\section{Trivalent CAR $T$ cells using the "OR" logic gate}

A trivalent CAR T-cell is a single engineered $T$ cell with three CARs targeting validated antigens. As shown by the data from the Meenakshi group above, in response to the complexity of the GBM microenvironment, the high heterogeneity of tumor cells and the low permeability of the blood-brain barrier, dual CAR-T cells co-targeting glioma-restricted antigens exhibited significant advantages in regard to inhibiting antigen escape and enhancing $\mathrm{T}$ cell activation and persistence [34]. However, due to the specific antigen pair variation between GBM patients, bispecific CAR-T cells still provide insufficient antigen coverage. Recently, trivalent peptide vaccines targeting glioma-restricted antigens, including EphA2, IL13r $\alpha 2$, and survivin, have been proven to be safe and feasible for the treatment of pediatric GBM [44]. Notably, CAR-T cell therapy can be unrestricted by the human leukocyte antigen (HLA), whereas the efficacy of vaccines has been hampered by the HLA. Therefore, to explore a more universal ACT for GBM patients, the Meenakshi group designed and validated trivalent CAR-T cells that simultaneously targeted HER2, IL13R $\alpha 2$, and EphA2, which achieved nearly $100 \%$ clearance of GBM tumor cells. This powerful antitumor effect of trivalent CAR-T cells in preclinical experiments is potentially due to enhanced transmission signal activation, expanded tumor antigen coverage and robust immune synapse formation [45] (Fig. 1c).

Despite broadening CAR-T cell applications, multiantigen-targeted strategies also carry risks associated with specific reduction and on-target, off-tumor toxicities. For these adverse reactions with the "OR" gated logic, the CAR-T cells modified with "AND" and "NOT" logic gate can be an optimal approach.

\section{"AND" and "NOT" logic-gated CAR-T cells for alleviating on-target, off-tumor toxicities Dual CAR-T cells using the "AND" logic gate} With the introduction of a costimulation signal domain into the CAR-T cell structure, second-generation CAR-
$\mathrm{T}$ cells exhibited long-term persistence and enhanced antitumor efficacy; however, they caused worse ontarget, off-tumor toxicities to normal tissues expressing low levels of the tumor-associated antigen (TAA). To address this problem, Lanitis et al. proposed a dual-signaling strategy that physically isolated signal 1 (CD3ל) from costimulatory signal 2 (CD28) and assembled these signals into separate CARs individually targeting mesothelin and afolate receptor (FRa). Signal 1 of the MSLN-targeted CAR

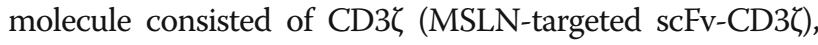
while signal 2 of the FRa-targeted CAR molecule contained the intracellular costimulatory domain of the CD28 (FRatargeted scFv-CD28). An "AND" logic gate was applied for dual-signaling CAR-T cells to achieve highly selective antitumor efficacy.

Low cytokine secretion levels were observed when dual-signaling CAR-T cells encountered cells expressing only a single TAA, whereas enhanced cytokine secretion was exhibited when cells co-expressing both antigens were targeted in vivo. Dual CAR-T cells showed antitumor activity and long-term persistence that were greater than first-generation CAR-T cells but was equal to second-generation CAR-T cells. Notably, the traditional second-generation CAR-T cells exhibited strong cytolytic effects toward tumor as well as toward normal cells expressing the only mesothelin, partially causing serious adverse reactions, whereas dual-signaling CAR-T cells showed selective specificity for tumor cells. Hence, this "AND" logic-engineered strategy allows dual-signaling CAR-T cells to exhibit the natural biological properties of $\mathrm{T}$ cells, such as optimized proliferation, cytokine secretion, cytotoxicity, tumor-specific homing and "offtumor" toxicity reduction [46] (Fig. 2a).

Recently, the "AND" logic-gated strategy has been advanced in an intelligent and customizable manner by employing a synthetic Notch receptor system, which regulates the expression of custom CAR in a transcriptional manner. Upon the binding of the first CAR (CAR1) to its cognate ligand, the SynNotch receptor is induced to release transcriptional regulators and to act in the nucleus to regulate the transcription and expression of the second CAR (CAR2). In the presence of the specific antigen targeted by CAR1, CAR2 is conditionally expressed; thus, these $\mathrm{T}$ cells are safely engineered to achieve the highly specific recognition and killing of tumor cells [47] (Fig. 2b).

\section{Trivalent CAR $T$ cells using the "AND" logic gate}

In general, the tumor microenvironment (TME) has strong immunosuppressive properties, including physical and immune barriers, which severely impede the antitumor response of CAR-T cells. Therefore, Sukumaran et al. generated a new type of trivalent CAR-T cell with an "AND" logic gate to overcome the immunosuppression 
of TME in a pancreatic cancer model. Rather than adding additional CARs targeting TAAs, the novel CAR-T cells respond to only tumor-specific expression patterns. The transforming growth factor (TGF $\beta$ ) and interleukin 4 (IL4), as widely accepted T cell immunosuppressive cytokines in TME, are potential targets. Hence, engineered $\mathrm{T}$ cell was redirected to recognize the tumor-specific antigen prostate stem cell antigen (PSCA) and immunosuppressive cytokines including TGF $\beta$ and IL4, thereby transmitting independent signals, which included antigen recognition, costimulation, and cytokine secretion. The synchronous combination of these three signals ultimately initiated $\mathrm{T}$ cell activation, amplification, and persistence, achieving safe, and selective cytolysis at tumor sites [48] (Fig. 2c).

\section{Dual CAR-T cells using the "NOT" logic gate}

The current mainstream approaches for eliminating the side effects of CAR-T cells are non-specific immunosuppression or lymphodepletion, such as high-dose corticosteroids, which are inhibitory or cytotoxicity to T cells, causing secondary complications and reducing the therapeutic efficacy [49].

In the field of adoptive cell therapy safety, the "NOT" logic gate is an effective complementary strategy to the "AND" logic gate. In a proof-of-concept study, Sadelain et al. designed an inhibitory CAR-T cell with distinct CAR molecules. One CAR contained a CD28/CD3 $\zeta$ signaling domain, which activated $\mathrm{T}$ cells to recognize

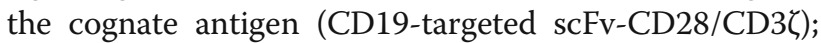
however, another CAR specific for prostate-specific membrane antigen (PSMA) consisted of an inhibitory signaling domain derived from PD-1 or CTLA-4 instead of a costimulatory domain (PSMA-targeted scFv-PD1/ CTLA-4). The expression of PSMA is mainly in metastatic prostate cancer but is also present in normal and benign tissues [50]. iCAR-T cells selectively kill tumor cells expressing CD19 molecules, whereas off-target cells co-expressing the CD19 and PSMA recognized by iCARs were protected from attack, allowing $\mathrm{T}$ cells to distinguish target cells from off-target cells. It is worth noting that iCAR-mediated inhibition is transient and reversible, similar to the inhibition mediated by natural killer cells. Despite previous exposure to inhibitory antigens, iCAR-T cells maintain reactivity against targeted antigens after detaching from inhibitory antigens. Several crucial factors profoundly influence the function of iCAR-T cells, and special attention will be required in the future clinical application phase, including toward the affinity and expression level of iCARs, appropriate target antigen selection and the optimization of the CAR/iCAR ratio [51] (Fig. 2d).

\section{Conclusions}

The clinical application of CAR $\mathrm{T}$ cell therapy has been limited by disease relapse caused by antigen escape and on-target, off-tumor toxicities due to low antigen specificity. To broaden the antigen coverage, $\mathrm{T}$ cells have been modified with multi-antigen targeting and combined with the "OR" logic gate to reduce antigen escape. Pooled, dual, tandem, and trivalent CAR-T cells with broader antigen coverage have been developed in preclinical and clinical trials. Because of the reduced antigen specificity, CAR-T cells with an "OR" logic gate could potentially have more severe on-target, off-tumor toxicities while reducing antigen escape. The "AND" and "NOT" logic gates better balance the relationship between antitumor efficacy and on-target, off-tumor toxicities. The SynNotch receptor system has applied the "AND" logic gate in an intelligent and customizable manner. In addition to adding additional chimeric antigen receptors, the "AND" logic gate can also provide engineered $\mathrm{T}$ cells with the ability to selectively respond to tumor-specific expression patterns. The antitumor efficiency of trivalent CAR T cells targeting PSCA, TGF $\beta$, and IL4 has been verified in a preclinical trial. The "NOT" logic gate enhanced the safety of CAR-T cells. Future multi-targeted antigen strategies require more optimized designs and more rational logic gates to expand the CAR-T cell therapeutic window.

\section{Abbreviations}

FDA: The US Food and Drug Administration; CAR-T: Chimeric antigen receptor-T; B-ALL: B cell acute lymphocytic leukemia; PD-1: Programmed death-1; CTLA-4: Cytotoxic T lymphocyte-associated protein-4; scFv: Singlechain variable fragment; ACT: Adoptive T cell therapy; CAIX: Carbonic anhydrase IX; CEA: Carcinoembryonic antigen; MSLN: Mesothelin;

IL13Ra2: Interleukin 13 receptor, alpha2; FAP: Fibroblast activation protein-a; ZFN: Zinc-finger nuclease; TCR: T cell receptor; HER2: Human epidermal growth factor receptor-2; CAA: Cholangiocarcinoma; EGFR: Epidermal growth factor receptor; PR: Partial remission; PET-CT: Positron emission tomographycomputed tomography; GBM: Glioblastoma; ZAP-70: Zeta-chain-associated protein kinase 70; VH: Variable heavy; VL: Variable light; R/R: Relapsed and refractory; HSCT: Hematopoietic stem cell transplantation; MRD: Minimal residual disease; CR: Complete remission; GVHD: Graft versus host disease; HLA: Histocompatibility antigen; TAA: Tumor-associated antigen; FRa: A-folate receptor; PSCA: Prostate stem cell antigen; PSMA: Prostate-specific membrane antigen; TGF $\beta$ : Transforming growth factor; IL4: Interleukin 4; GFP: Green fluorescent protein

\section{Acknowledgments}

Not applicable.

\section{Authors' contributions}

$\mathrm{XH}$ and WDH conceived and designed the study. XH, JSW, and YW collected and interpreted the data. $\mathrm{XH}$ and WDH wrote the manuscript. JSW and YW provided technical or material support. WDH supervised the study. All authors read and approved the final manuscript.

\section{Funding}

This research was supported by the grants from the National Natural Science Foundation of China (No. 81830002 and 31870873 to WDH) and the National Key Research and Development Program of China (No. 2016YFC1303501 and 2016 YFC1303504 to WDH, and No.2017YFC0909803 to WY) 


\section{Availability of data and materials}

All data generated or analyzed in this study are included in this article. Other data that are relevant to this article are available from the corresponding author upon reasonable request.

\section{Ethics approval and consent to participate}

Not applicable.

\section{Consent for publication}

No individual data were used in this study.

\section{Competing interests}

The authors declare that they have no competing interests.

Received: 13 September 2019 Accepted: 27 October 2019

Published online: 29 November 2019

\section{References}

1. Mullard A. FDA approves first CAR T therapy. Nat Rev Drug Discov. 2017; 16(10):669.

2. FDA Approves Second CAR T-cell Therapy. Cancer Discov 2018, 8(1):5-6.

3. Rizvi NA, Hellmann MD, Snyder A, Kvistborg P, Makarov V, Havel J, et al. Cancer immunology. Mutational landscape determines sensitivity to PD-1 blockade in non-small cell lung cancer. Science. 2015;348(6230):124-8.

4. Sadelain M, Riviere I, Riddell S. Therapeutic T cell engineering. Nature. 2017; 545(7655):423-31.

5. Dai H, Wang Y, Lu X, Han W. Chimeric antigen receptors modified T-cells for cancer therapy. J Natl Cancer Inst 2016, 108(7).

6. Maude SL, Laetsch TW, Buechner J, Rives S, Boyer M, Bittencourt H, et al. Tisagenlecleucel in children and young adults with B-cell lymphoblastic leukemia. N Engl J Med. 2018;378(5):439-48.

7. June $\mathrm{CH}$, O'Connor RS, Kawalekar OU, Ghassemi S, Milone MC. CAR T cell immunotherapy for human cancer. Science. 2018;359(6382):1361-5.

8. Krenciute G, Prinzing BL, Yi Z, Wu MF, Liu H, Dotti G, et al. Transgenic expression of IL15 improves antiglioma activity of IL13Ralpha2-CAR T cells but results in antigen loss variants. Cancer Immunol Res. 2017:5(7):571-81.

9. O'Rourke DM, Nasrallah MP, Desai A, Melenhorst JJ, Mansfield K, Morrissette JJD et al. A single dose of peripherally infused EGFRvIll-directed CAR T cells mediates antigen loss and induces adaptive resistance in patients with recurrent glioblastoma. Sci Transl Med 2017, 9(399).

10. Sommermeyer D, Hill T, Shamah SM, Salter Al, Chen Y, Mohler KM, et al. Fully human CD19-specific chimeric antigen receptors for T-cell therapy. Leukemia. 2017:31(10):2191-9.

11. Zhao Z, Condomines M, van der Stegen SJC, Perna F, Kloss CC, Gunset G, et al. Structural design of engineered costimulation determines tumor rejection kinetics and persistence of CAR T Cells. Cancer Cell. 2015;28(4): 415-28.

12. Kochenderfer JN, Dudley ME, Kassim SH, Somerville RP, Carpenter RO, Stetler-Stevenson $\mathrm{M}$, et al. Chemotherapy-refractory diffuse large B-cell lymphoma and indolent B-cell malignancies can be effectively treated with autologous T cells expressing an anti-CD19 chimeric antigen receptor. J Clin Oncol. 2015:33(6):540-9

13. Long $A H$, Haso WM, Shern JF, Wanhainen KM, Murgai M, Ingaramo M, et al. 1374-1BB costimulation ameliorates T cell exhaustion induced by tonic signaling of chimeric antigen receptors. Nat Med. 2015;21(6):581-90

14. Kawalekar OU, RS OC, Fraietta JA, Guo L, McGettigan SE, Posey AD, Jr. et al. Distinct signaling of coreceptors regulates specific metabolism pathways and impacts memory development in CAR T cells. Immunity 2016, 44(3):712.

15. Sotillo E, Barrett DM, Black KL, Bagashev A, Oldridge D, Wu G, et al. Convergence of acquired mutations and alternative splicing of CD19 enables resistance to CART-19 immunotherapy. Cancer Discov. 2015;5(12):1282-95.

16. Fischer J, Paret C, El Malki K, Alt F, Wingerter A, Neu MA, et al. CD19 Isoforms enabling resistance to CART-19 immunotherapy are expressed in B-ALL patients at initial diagnosis. J Immunother. 2017;40(5):187-95.

17. Fry TJ, Shah NN, Orentas RJ, Stetler-Stevenson M, Yuan CM, Ramakrishna S, et al. CD22-targeted CAR T cells induce remission in B-ALL that is naive or resistant to CD19-targeted CAR immunotherapy. Nat Med. 2018;24(1):20-8.

18. Hamieh M, Dobrin A, Cabriolu A, van der Stegen SJC, Giavridis T, MansillaSoto J, et al. CAR T cell trogocytosis and cooperative killing regulate tumour antigen escape. Nature. 2019;568(7750):112-6.
19. Zhang Y, Zhang W, Dai H, Wang Y, Shi F, Wang C, et al. An analytical biomarker for treatment of patients with recurrent B-ALL after remission induced by infusion of anti-CD19 chimeric antigen receptor T (CAR-T) cells. Sci China Life Sci. 2016:59(4):379-85.

20. Morgan RA, Yang JC, Kitano M, Dudley ME, Laurencot CM, Rosenberg SA. Case report of a serious adverse event following the administration of $T$ cells transduced with a chimeric antigen receptor recognizing ERBB2. Mol Ther. 2010;18(4):843-51.

21. Lamers $\mathrm{CH}$, Sleijfer $\mathrm{S}$, Vulto AG, Kruit WH, Kliffen M, Debets $R$, et al. Treatment of metastatic renal cell carcinoma with autologous Tlymphocytes genetically retargeted against carbonic anhydrase IX: first clinical experience. J Clin Oncol. 2006;24(13):e20-2.

22. Brown CE, Badie B, Barish ME, Weng L, Ostberg JR, Chang WC, et al. Bioactivity and Safety of IL13Ra2-redirected chimeric antigen receptor CD8+ T cells in patients with recurrent glioblastoma. Clin Cancer Res. 2015:21(18): 4062-72.

23. Katz SC, Burga RA, McCormack E, Wang $L$, Mooring W, Point GR, et al. Phase I hepatic immunotherapy for metastases study of intra-arterial chimeric antigen receptor-modified T-cell therapy for CEA+ liver metastases. Clin Cancer Res. 2015;21(14):3149-59.

24. Klampatsa A, Haas AR, Moon EK, Albelda SM. Chimeric antigen receptor (CAR) T cell therapy for malignant pleural mesothelioma (MPM). Cancers (Basel). 2017:9(9).

25. Kakarla S, Chow KK, Mata M, Shaffer DR, Song XT, Wu MF, et al. Antitumor effects of chimeric receptor engineered human T cells directed to tumor stroma. Mol Ther. 2013;21(8):1611-20.

26. Wang LC, Lo A, Scholler J, Sun J, Majumdar RS, Kapoor V, et al. Targeting fibroblast activation protein in tumor stroma with chimeric antigen receptor T cells can inhibit tumor growth and augment host immunity without severe toxicity. Cancer Immunol Res. 2014:2(2):154-66.

27. Tran E, Chinnasamy D, Yu Z, Morgan RA, Lee CC, Restifo NP, et al. Immune targeting of fibroblast activation protein triggers recognition of multipotent bone marrow stromal cells and cachexia. J Exp Med. 2013;210(6):1125-35.

28. Chmielewski M, Hombach A, Heuser C, Adams GP, Abken H. T cell activation by antibody-like immunoreceptors: increase in affinity of the single-chain fragment domain above threshold does not increase $T$ cell activation against antigen-positive target cells but decreases selectivity. J Immunol. 2004;173(12):7647-53

29. Park S, Shevlin E, Vedvyas Y, Zaman M, Park S, Hsu YS, et al. Micromolar affinity CAR T cells to ICAM-1 achieves rapid tumor elimination while avoiding systemic toxicity. Sci Rep. 2017;7(1):14366

30. Watanabe K, Kuramitsu S, Posey AD Jr, June $\mathrm{CH}$. Expanding the therapeutic window for CAR T cell therapy in solid tumors: the knowns and unknowns of CAR T Cell Biology. Front Immunol. 2018;9:2486

31. Varela-Rohena A, Carpenito C, Perez EE, Richardson M, Parry RV, Milone M, et al. Genetic engineering of T cells for adoptive immunotherapy. Immunol Res. 2008:42(1-3):166-81.

32. Osborn MJ, Webber BR, Knipping F, Lonetree CL, Tennis N, DeFeo AP, et al. Evaluation of TCR gene editing achieved by TALENS, CRISPR/Cas9, and megaTAL Nucleases. Mol Ther. 2016;24(3):570-81.

33. Wei J, Han X, Bo J, Han W. Target selection for CAR-T therapy. J Hemato Oncol. 2019;12(1):62

34. Hegde M, Corder A, Chow KK, Mukherjee M, Ashoori A, Kew Y, et al. Combinational targeting offsets antigen escape and enhances effector functions of adoptively transferred T cells in glioblastoma. Mol Ther. 2013;21(11):2087-101.

35. Ruella M, Barrett DM, Kenderian SS, Shestova O, Hofmann TJ, Perazzelli J, et al. Dual CD19 and CD123 targeting prevents antigen-loss relapses after CD19-directed immunotherapies. J Clin Invest. 2016;126(10):3814-26.

36. Zah E, Lin MY, Silva-Benedict A, Jensen MC, Chen YY. T Cells Expressing CD19/CD20 bispecific chimeric antigen receptors prevent antigen escape by malignant B cells. Cancer Immunol Res. 2016;4(6):498-508.

37. Feng KC, Guo YL, Liu Y, Dai HR, Wang Y, Lv HY, et al. Cocktail treatment with EGFR-specific and CD133-specific chimeric antigen receptor-modified T cells in a patient with advanced cholangiocarcinoma. J Hematol Oncol. 2017;10(1):4

38. Grada Z, Hegde M, Byrd T, Shaffer DR, Ghazi A, Brawley VS, et al. TanCAR: a novel bispecific chimeric antigen receptor for cancer immunotherapy. Mol Ther Nucleic Acids. 2013;2(7):e105.

39. Hegde M, Mukherjee M, Grada Z, Pignata A, Landi D, Navai SA, et al. Tandem CAR T cells targeting HER2 and IL13Ra2 mitigate tumor antigen escape. J Clin Invest. 2016;126(8):3036-52. 
40. Jensen MC, Riddell SR. Design and implementation of adoptive therapy with chimeric antigen receptor-modified T cells. Immunol Rev. 2014;257(1):127-44

41. Muyldermans S. Nanobodies: natural single-domain antibodies. Annu Rev Biochem. 2013:82:775-97.

42. De Munter S, Ingels J, Goetgeluk G, Bonte S, Pille M, Weening K et al. Nanobody based dual specific CARs. Int J Mol Sci 2018, 19(2).

43. Jia H, Wang Z, Wang Y, Liu Y, Dai H, Tong C, et al. Haploidentical CD19/ CD22 bispecific CAR-T cells induced MRD-negative remission in a patient with relapsed and refractory adult B-ALL after haploidentical hematopoietic stem cell transplantation. J Hematol Oncol. 2019;12(1):57.

44. Pollack IF, Jakacki Rl, Butterfield LH, Hamilton RL, Panigrahy A, Potter DM et al. Antigen-specific immune responses and clinical outcome after vaccination with glioma-associated antigen peptides and polyinosinicpolycytidylic acid stabilized by lysine and carboxymethylcellulose in children with newly diagnosed malignant brainstem and nonbrainstem gliomas. J Clin Oncol 2014, 32(19):2050-8.

45. Bielamowicz K, Fousek K, Byrd TT, Samaha H, Mukherjee M, Aware N, et al. Trivalent CAR T cells overcome interpatient antigenic variability in glioblastoma. Neuro Oncol. 2018;20(4):506-18.

46. Lanitis E, Poussin M, Klattenhoff AW, Song D, Sandaltzopoulos R, June CH, et al. Chimeric antigen receptor $T$ cells with dissociated signaling domains exhibit focused antitumor activity with reduced potential for toxicity in vivo. Cancer Immunol Res. 2013;1(1):43-53.

47. Roybal KT, Rupp LJ, Morsut L, Walker WJ, McNally KA, Park JS, et al. Precision Tumor Recognition by T Cells With Combinatorial Antigen-Sensing Circuits. Cell. 2016;164(4):770-9.

48. Sukumaran S, Watanabe N, Bajgain P, Raja K, Mohammed S, Fisher WE, et al. Enhancing the potency and specificity of engineered T cells for cancer treatment. Cancer Discov. 2018;8(8):972-87.

49. Akpek G, Lee SM, Anders V, Vogelsang GB. A high-dose pulse steroid regimen for controlling active chronic graft-versus-host disease. Biol Blood Marrow Transplant. 2001;7(9):495-502.

50. Wright GL Jr, Haley C, Beckett ML, Schellhammer PF. Expression of prostatespecific membrane antigen in normal, benign, and malignant prostate tissues. Urol Oncol. 1995; 1 (1):18-28,

51. Fedorov VD, Themeli M, Sadelain M. PD-1- and CTLA-4-based inhibitory chimeric antigen receptors (iCARs) divert off-target immunotherapy responses. Sci Transl Med 2013, 5(215):215ra172.

\section{Publisher's Note}

Springer Nature remains neutral with regard to jurisdictional claims in published maps and institutional affiliations.

Ready to submit your research? Choose BMC and benefit from:

- fast, convenient online submission

- thorough peer review by experienced researchers in your field

- rapid publication on acceptance

- support for research data, including large and complex data types

- gold Open Access which fosters wider collaboration and increased citations

- maximum visibility for your research: over $100 \mathrm{M}$ website views per year

At $\mathrm{BMC}$, research is always in progress.

Learn more biomedcentral.com/submissions 